\title{
PROPOSTA DE UM MODELO ESTRATÉGICO DE GESTÃO DE COMPRAS DE MATERIAIS E SERVIÇOS EM EMPRESA DE NUTRIÇÃO ANIMAL
}

\section{PROPOSAL FOR A STRATEGIC MODELFOR PURCHASE OF MATERIALS AND SERVICESIN ANUTRITIONCOMPANYANIMAL}

\author{
Alexsandro Monteiro \\ Novanis Animal LTDA - Rondonópolis - MT- Brasil \\ alexmonteiro9984@gmail.com \\ María Auxiliadora Cannarozzo Tinoco \\ Universidade Federal do Rio Grande do Sul - Porto Alegre - RS - Brasil \\ maria@producao.ufrgs.br
}

\begin{abstract}
Resumo
Em um cenário globalizado e competitivo, as empresas precisam rever seus modelos de gestão de compras, visto que as aquisições têm sido estratégicas para as organizações na redução de custos e aumento de sua competitividade, e para que isso ocorra é importante que as empresas adotem um modelo de compras estratégico adequado às suas necessidades. Neste contexto, este trabalho teve como objetivo, propor um modelo de gestão que torne o setor de compras da empresa em estudo, alinhado as suas estratégias utilizando como base os estudos realizados por Monteiro e Tinoco, (2013) e realizado as seguintes etapas: (i) Levantamento dos principais modelos de gestão estratégica de compras de materiais e serviços existentes na literatura; (ii) Verificação dos objetivos estratégicos e o alinhamento do processo de compras, devido a mudança na diretoria da empresa; (iii) Estruturação do modelo estratégico de gestão de compras da empresa em estudo e (iv) Validação do modelo proposto com a alta diretoria da empresa. Finalizadas estas etapas, obteve-se como resultado um modelo de gestão que alinha o setor de compras de materiais $e$ serviços aos objetivos estratégicos da empresa, apresentando as macro etapas do processo de compra estratégico, o fluxograma detalhado das etapas e o detalhamento das atividades em cada etapa do processo. Além disso, o modelo sugere a implementação de ferramentas de compras estratégicas adequadas ao perfil da empresa, no caso a Matriz de Kraljic, (1983) aliada ao modelo de Ogden (2003), ambas adaptadas ao contexto analisado.
\end{abstract}

Palavra-chave: Processo; compras; Gestão de compras.

\section{Introdução}

Atualmente a competição entre as empresas deixou de ser meramente entre unidades de negócios de forma isolada e passou a ser entre cadeias de suprimentos de forma integrada, fazendo com que a gestão eficiente de toda a cadeia de suprimentos permita torná-la competitiva (LAMBERT et al., 1998). 
A gestão de uma cadeia de suprimentos, engloba o planejamento e gerenciamento de todas as suas atividades no que diz respeito a aquisição e processamento da matéria-prima e entrega de um produto acabado ao consumidor final, por isso é preciso que haja a colaboração entre os membros da cadeia (ELLRAM; COOPER, 2014). Sendo assim, Barney(2012) e Souza; Scavarda;Scavarda(2008) ressaltam que as empresas participantes da cadeia, precisam integrar seus processos de gestão,inter e intra empresas dentro da cadeia, de forma a entregar valor aos clientes e garantir vantagem competitiva a todos os membros da cadeia.

Ainda sobre a gestão da cadeia de suprimentos, um ponto comum na literatura é a ênfase dada à otimização do processo de aquisição de materiais, principalmente, no que se refere à matéria-prima, o que justifica a importância que vem sendo dada ao setor de compras para que as empresas melhorem a sua competitividade (SOUZA; SCAVARDA; SCAVARDA, 2008; WEELE; RAAIJ, 2014).

Kraljic, (1983) complementa que uma empresa com a função compras, executada através de processos obsoletos, impactam na eficiência do setor e no relacionamento das empresas com seus parceiros da cadeia de suprimentos, e isso custa caro para a organização, por isso, os gestores de compras não devem ser indiferentes às mudanças e evolução nas práticas e técnicas de gestão de compras.

No Brasil, esta evolução no setor de compras iniciou-se com a crise do petróleo em 1970, desde então, as empresas passaram a dar uma atenção diferenciada e maior relevância ao setor de compras, tornando-o participante das formulações estratégicas da empresa (BRAGA, 2006).

SegundoGaither;Frazier (2001) isso vem acontecendo pelo fato de o setor de compras desempenhar um papel fundamental na realização dos objetivos estratégicos da empresa. Pois afetam elementos chaves na estratégia de operações de qualquer empresa como a entrega de produtos e serviços oferecidos pela organização no tempo certo, os custos das operações e a qualidade dos produtos e serviços (WEELE; RAAIJ, 2014).

Outro aspecto importante na determinação do setor de compras como um setor estratégico para a empresa, é que, se bem administrado, oferece grandes oportunidades de redução dos gastos na empresa, e consequentemente, isso acaba incrementando os seus lucros, pois cada unidade monetária economizada em compras gera mais uma unidade monetária de lucro (DIAS, 2003).

Sobre este ponto de vista, Andrade (2012), Sampson; Spring (2012) afirmam que a compra de materiais diretos, indiretos e os serviços contratados pelas empresas para o desenvolvimento de suas atividades cotidianas, têm forte implicação na sua competitividade, e isso por sua vez, consolida o setor de compras como estratégico nas organizações. Com o desenvolvimento de modelos e ferramentas que visam tornar a função compras mais eficaz e eficiente, esta consolidação 
do setor de compras nas empresas, tem se acelerado, porque vem proporcionando um maior alinhamento entre os objetivos estratégicos da empresa e a cadeia de suprimentos como um todo (KIBBELING, 2010; JOHNSEN; HOWARD; MIEMCZYK, 2014).

Dentre as ferramentas e técnicas de gestão estratégica de compras citadas na literatura, Bailyet al. (2000) sugerem a matriz de kraljic, como ferramenta que visa dar suporte ao departamento de compras para a seleção estratégica de materiais a serem adquiridos pela empresa, ao cruzar duas dimensões: o impacto sobre o resultado financeiro e a incerteza de oferta.

Neste contexto, o presente artigo objetiva propor um modelo de gestão de compras de materiais e serviços mais adequado às necessidades de uma empresa de nutrição animal, levando em consideração as suas estratégias e o uso das ferramentas para gestão de compras propostas na literatura.

\section{Referencial teórico}

A seguir é apresentada uma revisão de literatura sobre os aspectos que envolvem a gestão estratégica de materiais e serviços, modelos de compra estratégia e gestão de estoque, como aspectos importantes na administração estratégica de compras.

\subsection{Modelos de Compra Estratégica}

O primeiro modelo a ser abordado neste trabalho é o modelo proposto por Ogden (2003). Segundo o autor, o processo de compra estratégica utiliza sete passos para a redução do número de fornecedores da empresa, apresentados na Figura 1 a seguir.

Figura 1: Sete passos do processo de compra estratégicas de Ogden (2003)

\begin{tabular}{|c|c|c|c|c|c|c|}
\hline Passo 1 & Passo 2 & Passo 3 & Passo 4 & Passo 5 & Passo 6 & Passo 7 \\
\hline $\begin{array}{l}\text { Definir } \\
\text { Times }\end{array}$ & $\begin{array}{l}\text { Definir } \\
\text { Metas e } \\
\text { Objetivos }\end{array}$ & $\begin{array}{c}\text { Levantar } \\
\text { Informações } \\
\text { dos } \\
\text { fornecedores }\end{array}$ & $\begin{array}{c}\text { Elaborar } \\
\text { Proposta de } \\
\text { fornecimento }\end{array}$ & $\begin{array}{c}\text { Análise técnica } \\
\text { e } \\
\text { Negociação }\end{array}$ & $\begin{array}{l}\text { Divulgação } \\
\text { interna }\end{array}$ & $\begin{array}{l}\text { Validar } \\
\text { resultado }\end{array}$ \\
\hline
\end{tabular}

Fonte: Adaptado de Ogden (2003)

Descrito de forma suscinta, Ogden (2003) explica que é preciso formar um time multifuncional, composto por profissionais do setor de compras e pelos gerentes e responsáveis dos setores solicitantes da empresa,todos capacitados para desenvolver estratégias de seleção de fornecedores e as metas e objetivos para as aquisições. Em seguida faz-se necessário levantar o máximo de informações dos fornecedores para selecionar os mais capacitados em atender a demanda da empresa. O próximo passo, é elaborar uma solicitação de proposta de fornecimento, com base em cinco critérios: (i) Nível tecnológico; (ii) Nível de qualidade no suporte ao cliente; (iii) 
Qualidade do material ou serviço; (iv) Prazo de entrega do material ou serviço; e por fim, (v) Preço do material ou serviço. Mediante a proposta de cada fornecedor, o time faz a avaliação técnica e a equipe de compras entra em negociação com os fornecedores que apresentarem a proposta mais interessante para a empresa. Determinados os novos fornecedores da empresa, faz-se uma divulgação interna para que todos os colaboradores saibam sobreeles, e por fim, é preciso acompanhar e avaliar os resultados obtidos com esta negociação.

Ogden (2003) acredita que esta estratégia de compras traz mútuo benefíciospara a empresa e seus fornecedores. Dentre os benefícios conseguidos pela empresa estão: melhoria no controle de custos, maior controle e melhoria na qualidade; melhoria nos níveis de estoque, redução de custos e diminuição dos esforços na gestão de fornecedores. Para os fornecedores os ganhos são: aumento de sua receita pelo maior número de material vendido, o que proporciona a produção em escala gerando redução de custo, e isso, por sua vez, proporciona maior margem para negociação com outros clientes, tornando o fornecedor mais competitivo e consequentemente gera um incremento na sua carteira de clientes. Por estas vantagens estabelecidas para ambas as partes, Ogden (2003) afirma que esta estratégia de redução do número de fornecedores estabelece uma relação "ganhaganha" entre a empresa e seus fornecedores.

Outro modelo de compra estratégica é o apresentado por Kraljic (1983). Segundo Ferreira e Kharlamov (2012) na diversidade de modelos estratégicos de compras, este se tornou referência. Para os autores, o modelo não só permite obter uma melhor percepção sobre o poder de barganha nas negociações, como também a escolha da estratégia mais adequada nas aquisições, reduzindo assim os riscos expostos no marcado para as empresas.

Liew(2014), ressalta que o modelo leva em consideração dois critérios apresentados por Kraljic (1983) como sendo essenciais para a definição de uma estratégia de compras para uma empresa, o impacto do material nas finanças e a dificuldade de aquisição do material. O resultado da relação entre estes fatores geram quatro categorias nas quais são classificados os materiais: (i) Itens não críticos; (ii) Itens gargalo; (iii) Itens alavancados e (iv) Itens estratégicos. A seguir apresenta-se a Figura 2 para melhorar o entendimento da matriz proposta por Kraljic (1983). 
Figura 2: Matriz de portifólio de Kraljic

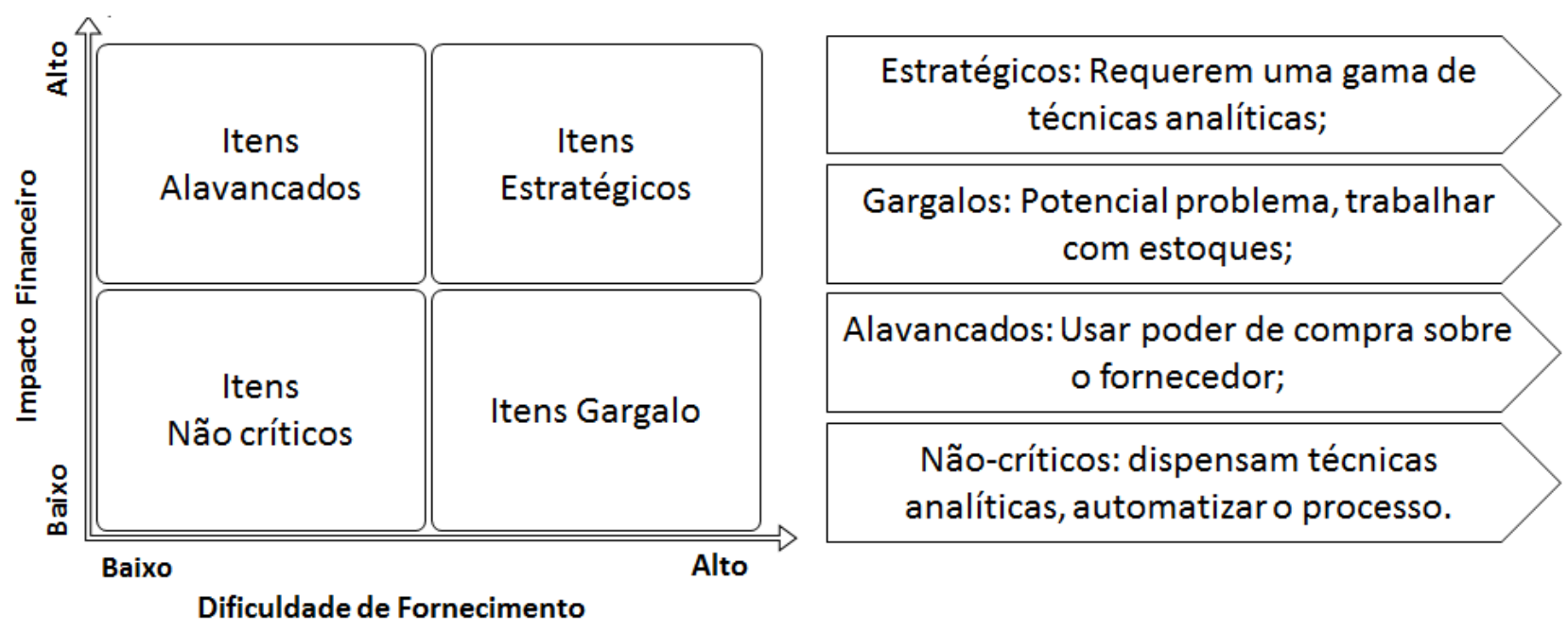

Fonte: Adaptado de Kraljic (1983)

Kraljic (1983) complementa sobre seu modelo de compra estratégica, que os materiais classificados em cada uma destas categorias, Não Críticos, Alavancados, Gargalos e Estratégicos, são abordados de forma diferente, e o nível de complexidade para cada uma delas está relacionado ao tipo de estratégia adotada pela empresa para cada categoria.

Os itens estratégicos pela sua alta influência nos resultados da empresa e sua grande complexidade de fornecimento, requerem uma gama de técnicas analíticas incluindo a análise de mercado, análise de risco, uso de programas de simulação e modelos de otimização, a previsão do preço, e vários outros tipos de análise microeconômica.

Por ser um potencial problema para a empresa por suas caraterísticas específicas, os itens gargalos também requerem técnicas de análises e estratégias bem elaboradas como o uso de estoque de segurança, monitoramento constante do uso destes itens e, por fim, a empresa deve precaver-se com o uso de planos de contingência de fornecimento.

Para os itens alavancados, a empresa já tem maior de poder de barganha sobre o fornecedor que permite explorar plenamente seu poder de compra, e pode fazer uso da concorrência entre fornecedores, estabelecer preços mínimos ou até mesmo a substituição de produtos, tudo isso, pela baixa complexidade de fornecimento.

Os itens não-críticos dispensam muitas das técnicas analíticas usadas nas outras categorias, as tomadas de decisões por exemplo são descentralizadas, pois tanto a sua disponibilidade quanto o seu valor não impactam nos resultados da empresa. Para esta categoria é comum a utilização de um modelo de otimização de estoques, garantia de volume de compra aos fornecedores e a padronização de produtos (KRALJIC,1983). 
À primeira vista, parecem recomendações bastante lógicas, no entanto, em uma análise mais aprofundada na natureza destas recomendações estratégicas, conclui-se que essas estratégias são bastante genéricas, proporcionando apenas um norte para o desenvolvimento das estratégias de compras mais adequadas para cada empresa, e assim, cada organização pode reagir e adaptar-se às circunstâncias existentes dentro da sua cadeia de abastecimento (GELDERMAN e WEELE, 2001). Ferreira e Kharlamov (2012) complementam que cada organização deve ajustar a matriz a sua realidade, apontando criteriosamente seus pontos críticos.Apesar das críticas, Gelderman e Donald (2008) apontam que o modelo de Kraljic tem sido abordado em muitas indústrias.

\subsection{Gestão de estoque}

De acordo com cada empresa, o estoque, que é o armazenamento de uma determinada quantia de material, tem valor, finalidade e administração diferente. Sua principal finalidade é evitar perdas de vendas por falta de produtos devido a interrupção na produção, e também servir como segurança para os casos como: perdas, oscilações na produção, quebra de máquinas, falta de funcionário e vendas imprevistas (SLACK et al, 1999; VIANA, 2002).

Para uma boa gestão de estoque é necessário levar em consideração dois fatores, a política de estoque e os custos remanescentes da manutenção do estoque na empresa (WANKE, 2011). Entretanto,Bowersox; Closs (2009) afirmam que a política de estoque é muito importante para a gestão do mesmo, pois está relacionada às normas estipuladas pela empresa sobre o que produzir ou comprar, quando, e em quais quantidades, tendo em vista minimizar o custo de manutenção de estoque sem afetar o nível de qualidade de atendimento ao cliente.

Para a gestão do estoque a classificação $\mathrm{ABC}$ dos materiais é uma ferramenta frequentemente utilizada em ambiente empresarial com base na teoria de Pareto, onde os esforços para controle do estoque, são concentrados em $20 \%$ dos itens que representam cerca de $80 \%$ do custo, denominados materiais A.Entretanto,Klippel et al. (2007) acrescentam que o método ABC, embora muito útil na gestão de estoque, apresenta algumas limitações, que no âmbito global da gestão de materiais pode trazer distorções perigosas para a empresa, uma vez que não considera a criticidade do material para o processo, mas apenas o seu valor monetário, por este motivo criou-se método ABC com criticidade (ANDRADE, 2012).

Dobler; Burt (1996) determinam esta criticidade operacional, através de uma escala onde: 1 - crítica; 2 - média; e 3: - não-crítica. Por esta escala apresentada pelos autores, um material A menos importante poderia ser tratado como sendo um item A-3, enquanto um material C crítico poderia ser identificado como sendo $\mathrm{C}$-1 e por meio desta classificação com dois dígitos permite 
que um material C-1 possa ter mais importância do que um material A-3 devido a sua criticidade, o que não seria possível adotando a tradicional classificação $\mathrm{ABC}$.

\section{Procedimentos metodológicos}

Para a realização deste trabalho, adotou-se como procedimento de pesquisa o estudo de caso. Yin (1989) afirma que o estudo de caso é uma inquirição empírica que investiga um fenômeno contemporâneo dentro de um contexto da vida real, quando a fronteira entre o fenômeno e o contexto não é claramente evidente e onde múltiplas fontes de evidência são utilizadas.

O presente artigo dá continuidade ao trabalho desenvolvido por Monteiro; Tinoco (2013), onde foi verificado o alinhamento entre o modelo de gestão de compras do setor de materiais e serviços e as políticas estratégias em uma empresa de nutrição animal. Nesse estudo constatou-se que existem etapas críticas do processo que não estão alinhadas às necessidades estratégicas devido, principalmente, à carência de uma estrutura adequada para realização das atividades conforme esperado. A necessidade de melhoria no processo é reconhecida pelos funcionários do setor, entretanto, é limitada a estrutura disponibilizada para executarem o processo de aquisição de bens materiais e serviços (MONTEIRO; TINOCO, 2013).

Conforme os autores, as etapas do processo diagnosticado, que tornam a gestão adotada pela empresa desalinhada com a política estratégica, e que precisam ser ajustadas são: Solicitação de compra e cotação; Processo de contratação de serviço; A entrega do material ao solicitante e Processamento da NotaFiscal (MONTEIRO; TINOCO, 2013).

Contudo, a empresa analisada passou por um período de mudanças na diretoria, indicando a possibilidade de novas orientações estratégicas para o setor de compras de materiais e serviços. Assim, surgiu a necessidade de uma reavaliação do alinhamento do modelo atual de compras, de forma a confirmar as etapas críticas e identificar novos aspectos que passaram a ser relevantes para a nova gestão da empresa, os quais devem ser incorporados no modelo de gestão de suprimentos a ser proposto.

Desta forma, para levar a cabo os objetivos propostos, o método de trabalho foi estruturado nas seguintes etapas:

- Levantamento dos principais modelos de gestão estratégica de compras de materiais e serviços existentes na literatura, a partir da revisão de estudos da literatura sobre modelos de gestão de compras e compra estratégica, onde destacaram-se os seguintes autores entre outros: e.g., Petrônio e Alt, 2009; Baily et al.,2000; Arnold, 1999; Monczka et al., 2011; Moreira, 2013; Ogden, 2003; Kraljic, 1983. 
- Verificação do alinhamento do processo de compras com os objetivos estratégicos da empresa, uma vez que a alta direção da empresa passou por mudanças na sua estrutura organizacional. A verificação foi realizada a partir de entrevistas com a diretoria da empresa, com base no questionário aplicado por Monteiro;Tinoco (2013). Foi entrevistada a Diretoria Administrativa Financeira que atualmente é responsável pelo setor de materiais e serviços, utilizando o mesmo questionário aplicado por Monteiro; Tinoco (2013) no intuito de verificar, dentro da mesma visão proposta pelos autores, se não houve mudança de estratégia.

- Estruturação do modelo estratégico para a gestão de compras de materiais e serviços para a empresa em estudo, a partir do mapeamento do macro processo e do processo detalhado de compra de materiais e serviços, considerando aspectos relevantes levantados na literatura sobre gestão estratégica de compras e as etapas críticas não alinhadas às necessidades estratégicas da empresa. Para o mapeamento do macro processo foi utilizada a ferramenta SIPOC (do inglês, Suppliers, Inputs ,Process, Outputs, Customers). O mapeamento detalhado do processo de compras, que foi realizado por meio de fluxograma padrão ANSI com auxílio do software Bizagi. Este fluxograma mostra todas as atividades e informações que ocorrem durante o processo de compra de materiais e serviços, as conexões entre as atividades e os movimentos que circundam cada atividade, usando como ponto de partida o diagrama SIPOC. Finalmente o modelo de compras de materiais e serviços envolve, além do mapa do processo (macro e detalhado), o uso de ferramentas de gestão estratégica de compras e de gestão de estoque.

- Validação do modelo proposto com a alta diretoria da empresa. Para isso, foi realizada uma entrevista individual com a diretoria Administrativa Financeira da empresa de forma a apresentar o modelo proposto para aprovação, validação e liberação para implantação. O modelo proposto foi apresentado ao entrevistado e foram incorporadas sugestões e melhorias.

\section{Análise e discução dos resultados}

Esta etapa do trabalho contempla a análise e discussão dos resultados da pesquisa realizada, abordando a proposição do modelo de gestão de compras estratégica de materiais e serviços mais adequado para uma empresa de nutrição animal, considerando o uso ferramentas propostas na literatura e as particularidades da empresa em estudo.

\subsection{Verificação do alinhamento do modelo atual com os objetivos estratégicos}

A verificação do alinhamento entre as necessidades estratégicas da empresa com o processo atual de compras, mostrou concordância entre os aspectos estratégicos apontados pela diretoria 
anterior e a nova diretoria administrativa, contudo, a nova diretoria indicou como aspecto estratégico, a gestão de estoque de materiais não produtivos, o qual não havia sido apontado no estudo de Monteiro; Tinoco (2013).

Segundo a diretoria atual, os estoques precisam ser gerenciados de maneira que sirva de ferramenta de apoio na gestão de compras de materiais não produtivos, que tem por objetivo principal não deixar que processos críticos de produção sejam interrompidos por falta de material, que muitas vezes são baratos, mas quando faltam, afeta diretamente no faturamento da empresa por deixar de produzir e atender a demanda de seus clientes. Desta forma, a gestão do estoque foi considera uma etapa crítica do processo a ser incorporado no modelo proposto.

O resultado da verificação do alinhamento do processo de compras com os objetivos estratégicos da empresa é apresentado na Tabela 1.

Tabela 1: Necessidade para alinhamento estratégico das etapas de compras.

\begin{tabular}{|c|c|c|}
\hline Etapas do processo & Alinhado? & Necessidade para alinhamento \\
\hline Solicitações de compras & Não & $\begin{array}{l}\text { Para as seis primeiras etapas do processo a seguir, as necessidades } \\
\text { para alinhamento são as mesmas. É preciso um sistema adequado }\end{array}$ \\
\hline $\begin{array}{l}\text { Recebimento da solicitação de } \\
\text { compras }\end{array}$ & Não & $\begin{array}{l}\text { que permita fazer as solicitações no mesmo software em que são } \\
\text { feitas as cotações e aprovações, assim, o setor de compras toma } \\
\text { conhecimento da aprovação da solicitação no mesmo instante em }\end{array}$ \\
\hline Planejamento de compra & Não & $\begin{array}{l}\text { que ela ocorrer, proporcionando assim, um melhor monitoramento } \\
\text { das compras. Com isso, também é possível fazer o planejamento de }\end{array}$ \\
\hline Solicitação de cotações & Não & $\begin{array}{l}\text { - compra de acordo com o caráter de cada solicitação, normal, urgente } \\
\text { ou emergencial. Pois, assim como a diretoria espera, a lista de } \\
\text { materiais a cotar é gerada já dentro do sistema no ato da solicitação. }\end{array}$ \\
\hline Determinação do preço & Parcial & $\begin{array}{l}\text { Depois de enviada a lista de material ao fornecedor e recebida a } \\
\text { cotação, o gerente da área realiza a aprovação via sistema de acordo }\end{array}$ \\
\hline $\begin{array}{l}\text { Emissão da ordem de compra } \\
\text { (OC) }\end{array}$ & Não & $\begin{array}{l}\text {-com sua alçada, e só então, o comprador gera a ordem de compra e } \\
\text { envia ao fornecedor escolhido. }\end{array}$ \\
\hline $\begin{array}{l}\text { Recepção e aceitação da } \\
\text { mercadoria }\end{array}$ & Não & $\begin{array}{l}\text { Para alinhar esta etapa, faz-se necessário uma redefinição do } \\
\text { processo atual, onde cada ator participante do processo, saiba } \\
\text { exatamente a sua responsabilidade. Para a diretoria é preciso uma } \\
\text { conferência adequada e a correta destinação do material. }\end{array}$ \\
\hline $\begin{array}{l}\text { Finalização da compra e } \\
\text { processamento da Nota Fiscal }\end{array}$ & Não & $\begin{array}{l}\text { A adequação do software e redefinição do processo refletem para o } \\
\text { alinhamento desta etapa. Segundo a diretoria, esta fase tem que ser } \\
\text { simples, com pouco lançamentos e trabalhos manuais. O software e } \\
\text { o processo precisam garantir a automação destas atividades. }\end{array}$ \\
\hline Contratação de serviços & Não & $\begin{array}{l}\text { Necessário um software adequado que possibilite tratar a contratação } \\
\text { de serviços como a compra de um material, respeitando as suas } \\
\text { particularidades que é no recebimento do serviço pelo solicitante, o } \\
\text { restante do processo é igual ao de compra de material. }\end{array}$ \\
\hline Gestão do estoque de materiais & Não & $\begin{array}{l}\text { Faz-se necessário para alinhamento ter também um software } \\
\text { adequado, e um processo bem definido das tarefas da função } \\
\text { compras, de forma que garanta uma acurácia do estoque de pelo } \\
\text { menos } 95 \% \text {. }\end{array}$ \\
\hline
\end{tabular}

Fonte:Pesquisa de campo(2014) 


\subsection{Proposta do modelo de compras de materiais e serviços}

O modelo de gestão de compras de materiais e serviços, proposto para a empresa analisada, está estruturado em dez etapas, fundamentadas nos estudos de Petrônio e Alt (2009); Baily et al. (2000); Arnold (1999); Monczka et al. (2011); Moreira (2013) e nas necessidades da empresa em estudo. As etapas do modelo são melhor detalhadas e explicadas através de: (i) o diagrama do macroprocesso (diagrama SIPOC); (ii) o fluxograma do processo e (iii) a descrição das atividades, os quais são apresentadas a seguir.

\subsubsection{Macroprocesso do modelo de gestão de compras de materiais e serviços}

A Figura 3, a seguir, apresenta o macroprocesso de gestão de compra de materiais e serviços do modelo, indicando os fornecedores, entradas, saídas e clientes, a partir do diagrama SIPOC (Supplier, Input, Process, Output, Customeer), no intuito de melhor visualizar as etapas do processo do modelo para que se perceba os elementos envolvidos no processo, e facilite a elaboração e compreensão do mapeamento detalhado do processo compras de materiais e serviços.

Figura 3: Estratégias de compras por categoria da Matriz de Kraljic (1983)

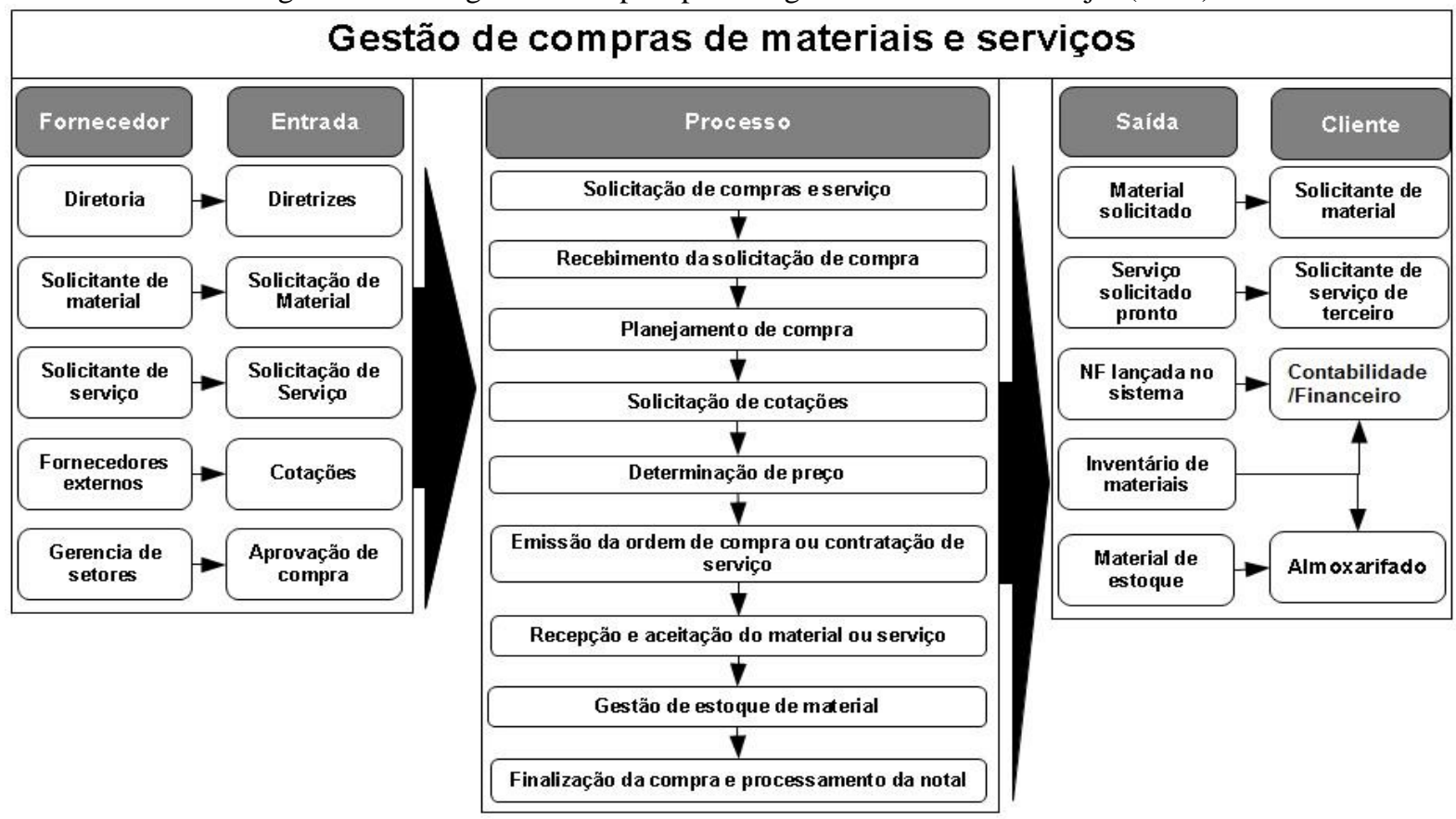

Fonte: Pesquisa de campo(2014)

Para melhor compreender o SIPOC, faz-se necessário definir os elementos do diagrama. O primeiro elemento do diagrama, os fornecedores, são os solicitantes de materiais e serviços, diretores da empresa, Fornecedores externos e Gerentes de setores. É importante ressaltar que no modelo de gestão de compras proposto, existem dois tipos de solicitantes, (i) os solicitantes de materiais e de serviços usuários, que são todas as pessoas que necessitam de material e/ou serviço 
para realização de suas atividades e (ii) solicitantes do sistema, que são pessoas autorizadas a solicitar no sistema, a compra de materiais ou serviços solicitados pelos usuários, por serem nomeados em cada setor pela alta gestão da empresa. Estes também são responsáveis por filtrar as solicitações dos usuários que serão ou não inseridas no sistema, dependendo da autorização do gestor responsável pela área que neste modelo proposto denomina-se aprovador.

O segundo elemento, as entradas, são as diretrizes por parte dos diretores, as solicitações de materiais e serviços emanadas dos solicitantes do sistema, as cotações dos fornecedores externos para determinação do preço de compras, e as aprovações por parte dos gestores da empresa das cotações que apresentam as melhores condições de compra, dentre as apresentadas pelos compradores. No terceiro elemento acontece o processamento de todas estas entradas para atender os clientes do setor de materiais e serviços.

Este processo realizado no terceiro elemento do SIPOC, consiste basicamente na cotação realizada pelos compradores dos materiais e serviços solicitados, em pelo menos três fornecedores previamente selecionados, em seguida o aprovador, gerente de setores, seleciona as melhores cotações para determinar o preço de compra e finalmente o comprador emite e envia ao fornecedor que ofereceu a melhor opção de compra para empresa, a Ordem de Compra ou Ordem de Serviço a Terceiro.

Seguindo as etapas do macro processo o almoxarife confere e recebe o material, e quando for prestação de serviço, o solicitante usuário confere e aceita o serviço. Estando tudo certo nas conferências, o almoxarife realiza a entrada dos dados da nota fiscal no sistema quando for material, pois a nota fiscal segue junto com ele, e se for serviço, depois do solicitante usuário aceitá-lo o solicitante do sistema libera para o comprador pedir a emissão da nota fiscal ao fornecedor e assim que recebê-la o almoxarife também realiza a entrada dos dados da nota fiscal no sistema, e por fim, em ambos os casos a nota fiscal fica liberada no sistema para ser contabilizada e paga. Também nesta etapa realiza-se a gestão dos materiais em estoque pelo almoxarife, principalmente.

Terminado o processo, passa-se então ao quarto elemento do diagrama, as saídas que são os resultados do processo no terceiro elemento do SIPOC: os materiais e serviços entregues ao solicitante usuário; as notas fiscais oriundas destas compras e/ou contratações de serviço, os inventários oriundos da gestão de estoque; e finalizando têm-se os materiais de estoque que ficam armazenados no almoxarifado para uso futuro. Geralmente as saídas do processo são o termômetro do processo, ou seja, a qualidade das saídas indica se o processo está sendo bem executado ou não.

Finalizando o SIPOC, tem-se o quinto elemento, os clientes, que para o processo de compras de materiais e serviços proposto, são: os solicitantes usuários, que recebe os materiais e serviços tal qual como solicitado; os departamentos de contabilidade e financeiro da empresa, que recebe a nota 
fiscal pronta para ser contabilizada e paga; o almoxarifado que recebe, todo material a ser estocado; e por fim o setor de contabilidade e o almoxarifado recebem os inventários, para o almoxarifado serve como ferramenta de apoio na gestão do estoque e para a contabilidade é usado para o cumprimento as exigências legais oriundas da modalidade contábil da empresa. Dando continuidade ao modelo de gestão proposto apresenta-se a seguir a próxima etapa do modelo que é a apresentação dos fluxogramas construído a partir do diagrama SIPOC.

\subsubsection{Fluxograma do modelo de gestão de compras de materiais e serviços}

As etapas do macro processo de compras foram desdobradas e melhor detalhadas, em micro atividades, passando por pontos de decisão, para a construção do fluxograma que representa o processo de compras detalhado.O fluxograma da Figura 04 representa o processo para solicitação de material e serviços que se inicia na necessidade do solicitante do material ou serviço e se estendendo até a finalização da solicitação no sistema. A Figura 5 por sua vez, representa o processo a ser utilizado pelo modelo para determinar o preço de compra e emissão da ordem de compra, dando sequência ao processo de solicitação. Este processo inicia-se na etapa subsequente a última etapa para solicitar material e serviço, que é verificar solicitações abertas, e termina na conferência e envio da Ordem de Compra ao fornecedor. Por fim na Figura 6, tem-se a representação do processo para receber e entregar o material ao solicitante, iniciando no monitoramento no sistema do material a chegar e finaliza no encaminhamento da nota fiscal a contabilidade, e assim conclui-se o processo de compra de materiais e serviços. 
Figura 4: Fluxograma do processo para solicitação de material e serviços

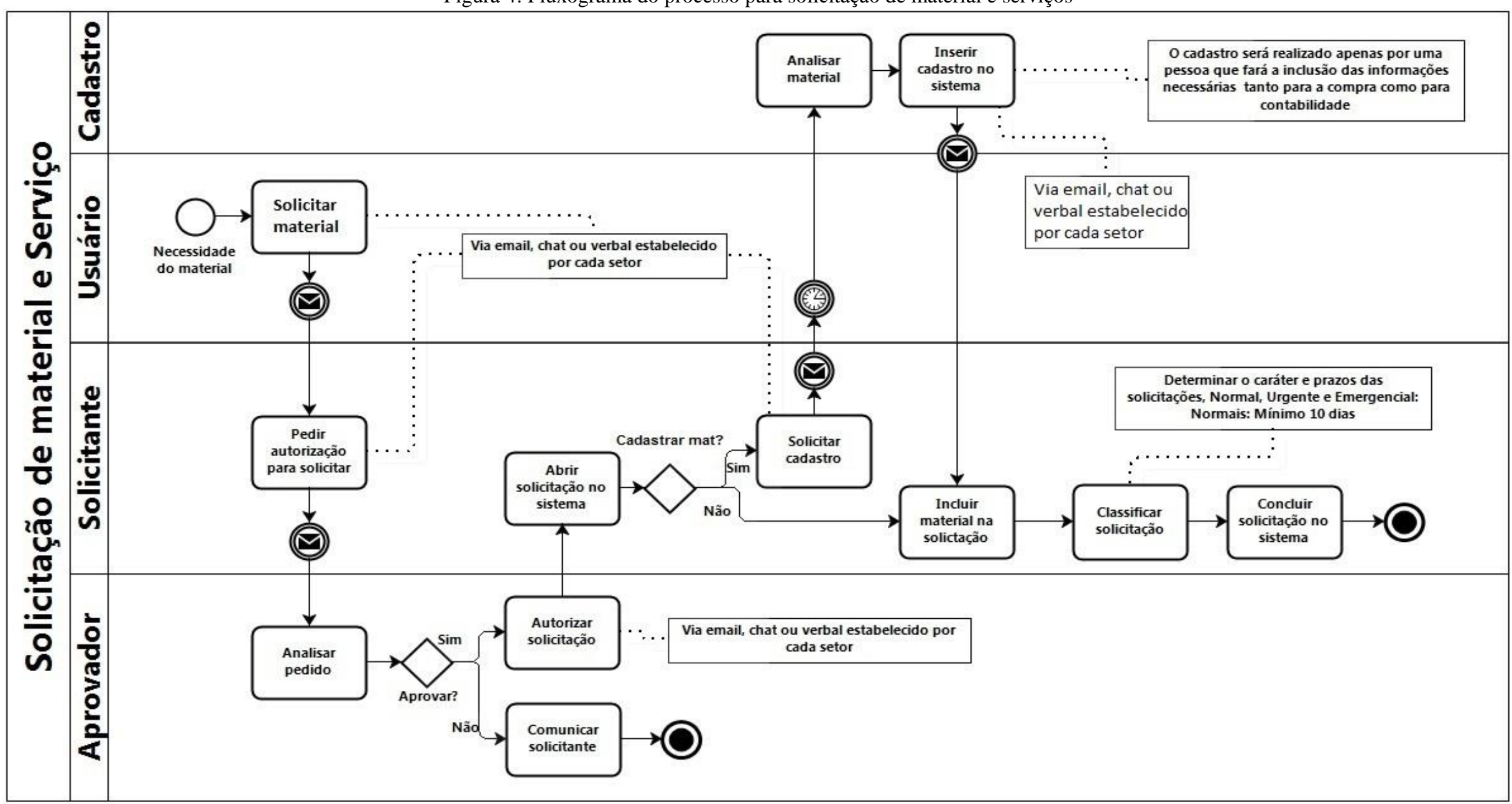

Fonte: Pesquisa de campo(2014) 
Figura 5: Fluxograma do processo para determinar o preço de compra a emissão, conferência e envio da Ordem de Compra

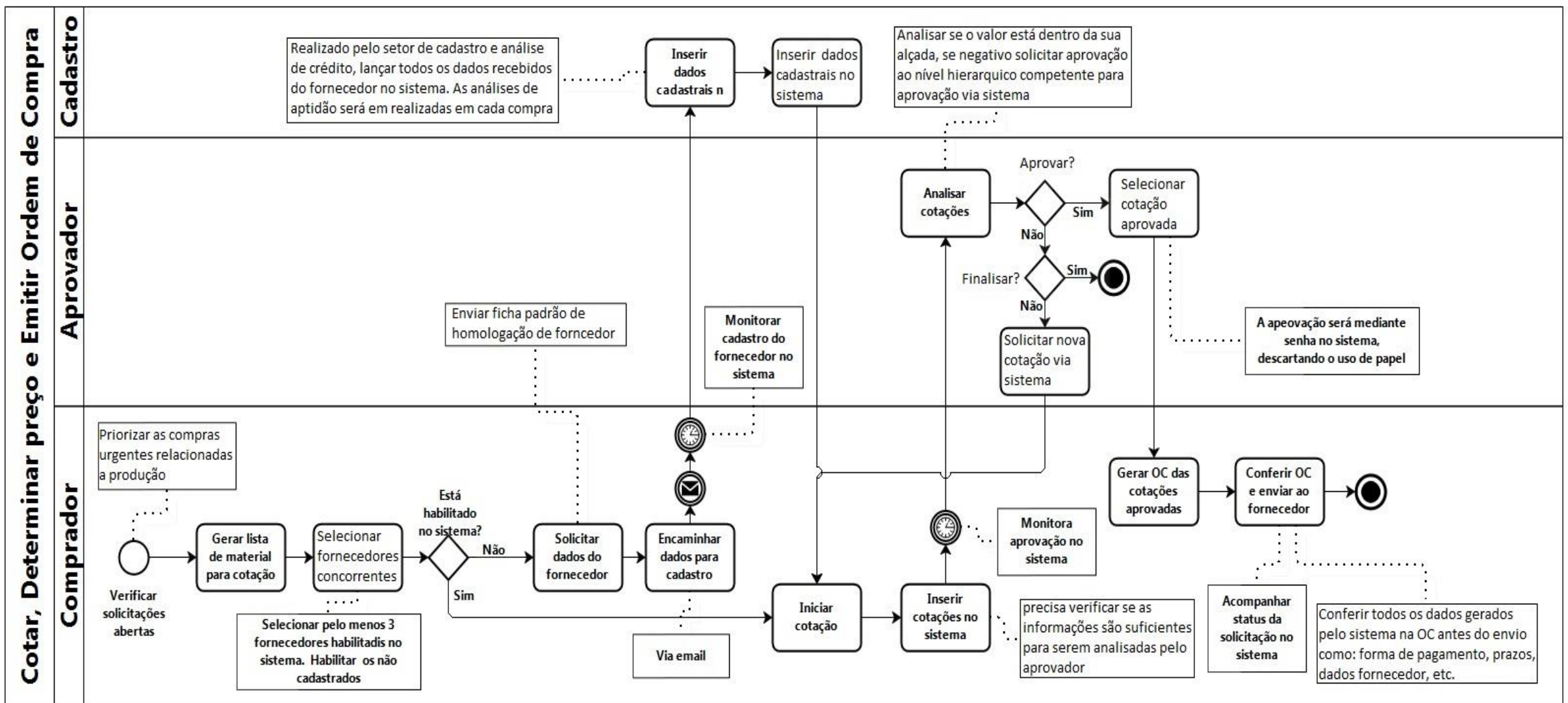

Fonte: Pesquisa de campo(2014) 
Figura 6: Fluxograma do processo para receber, conferir e entregar o material ao solicitante, e nota fiscal a contabilidade.

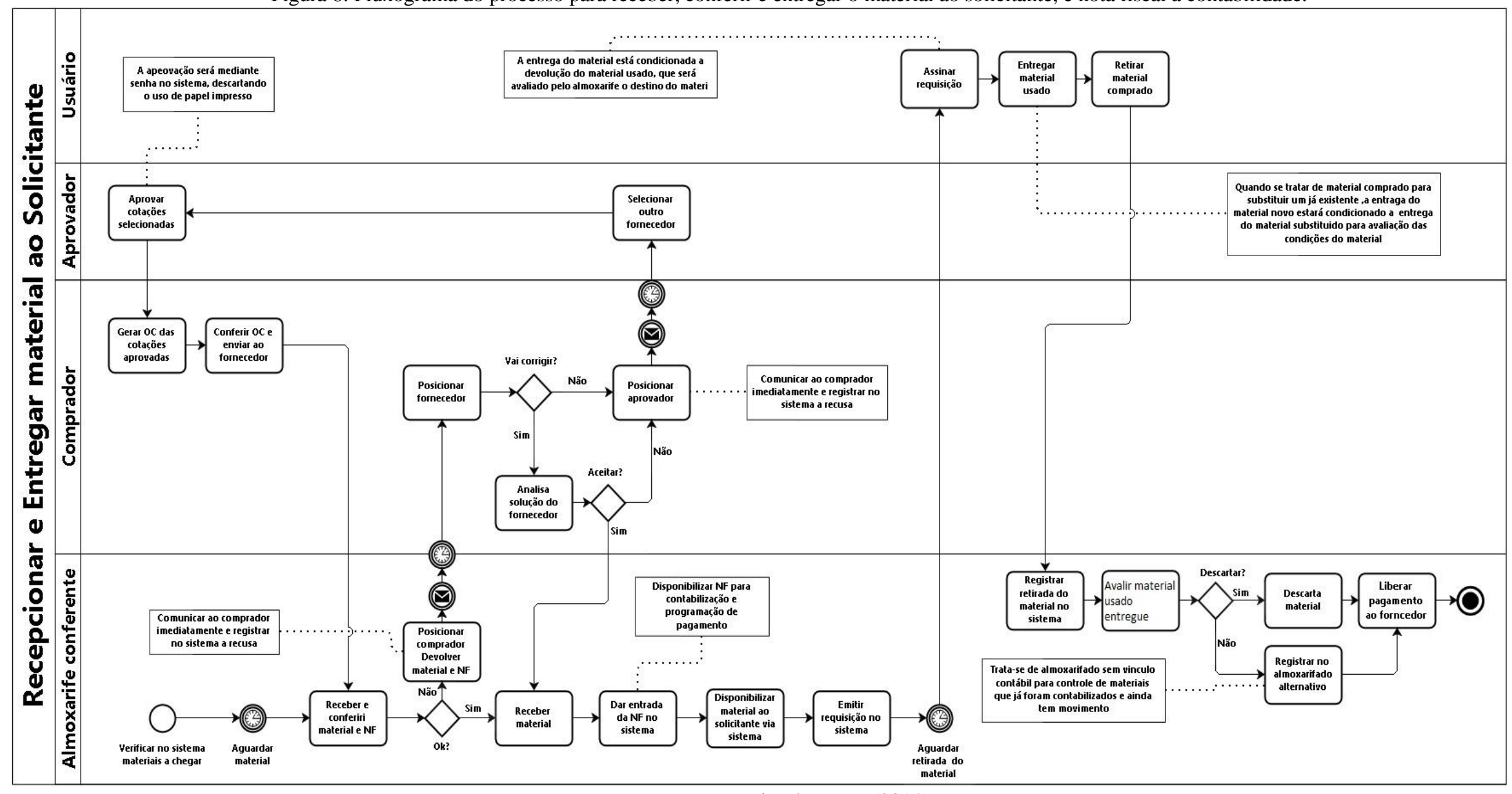

Fonte: Pesquisa de campo(2014) 


\subsubsection{Ferramentas para a gestão do estoque}

Na etapa de gestão de estoque (etapa 10 do modelo), propõe-se o uso de ferramentas adequadas para aquisição de materiais de baixos valores. Neste caso, sugere-se trabalhar com a ferramenta proposta por Ballou (2006) de estoque máximo e mínimo. Ao utilizar esta ferramenta, o sistema realiza a solicitação automática de ressuprimento do estoque quando o material chegar ao seu limite mínimo para atingir o estoque máximo do material, que é a quantidade que deve servir como estoque objetivo no momento em que se coloca um pedido.

Optou-se por utilizar esta ferramenta ao verificar nos históricos de inventário da empresa que nunca tiveram uma acurácia maior que cinquenta por cento, caracterizando com isso, um estoque errático e irregular quanto ao seu controle. Registros de compras da empresa, analisados no estudo, mostram que a empresa compra muitos materiais de baixo valor agregado, são mais de 5.000 tipos de itens cadastrados, o que torna a aplicação de ferramentas mais elaborada, morosa e com poucas vantagens para empresa do ponto de vista financeiro. Entretanto, a falta de estoque de alguns desses milhares de itens, apesar de baratos, coloca a produção em risco, tornando-se assim, itens críticos que necessitam de controle.

Mediante o exposto, optou-se por utilizar também, a classificação ABC dos materiais com criticidade, para concentrar esforços nos materiais mais caros e/ou críticos para a empresa. Para obter a classificação $\mathrm{ABC}$ com criticidade foi preciso avaliar a criticidade dos materiais de acordo com critérios estabelecidos, levando-se em consideração a importância operacional do item.

Para classificar os itens em A, B ou C, seguiu-se os seguintes passos: (i) Relacionar os itens de estoque; (ii) mensurar o valor total do item no estoque; (iii) ordenar os itens do estoque pelo seu valor total em ordem decrescente; (iv) determinar o percentual de representatividade do valor total de cada item no valor total do estoque; (v) classificar os itens do estoque como A, B ou C.Os resultados destas classificações são apresentados nas figuras 7 e 8 a seguir. 
Figura 7: Avaliação da criticidade dos materiais da empresa

\begin{tabular}{|c|c|c|c|c|c|}
\hline Material & Unid. & $\begin{array}{l}\text { Preço } \\
\text { médio }\end{array}$ & C1 & C2 & C3 \\
\hline Gás GLP 20kg & Un & 77,00 & $\mathbf{x}$ & & \\
\hline Respirador PFF2 & Un & 0,86 & & $\mathbf{x}$ & \\
\hline Papel toalha $6 \times 20 \times 200 \mathrm{mts}$ & rolo & 13,61 & & & $\mathbf{x}$ \\
\hline Sacos de amostras & Un & 0,35 & & & $\mathbf{x}$ \\
\hline Redutor M 8 - 1/15 & Un & $1.702,19$ & $\mathbf{x}$ & & \\
\hline Celula de carga modelo $\mathrm{S}$ & peca & 689,89 & & $\mathbf{x}$ & \\
\hline Redutor M $10-1 / 15$ & peca & $2.320,55$ & $\mathbf{x}$ & & \\
\hline 1331 - peça máquina de costura & peca & 239,20 & $\mathbf{x}$ & & \\
\hline Disco de corte $4.1 / 2^{\prime \prime} \times 1 / 16 \times 7 / 8$ & peca & 3,57 & & & $\mathbf{x}$ \\
\hline 1314 - peça máquina de costura & peca & 437,36 & $\mathbf{x}$ & & \\
\hline Touca TNT & peca & 0,09 & & & $\mathbf{x}$ \\
\hline Adesivo p/ Correia $1000 \mathrm{~g}$ & frasc & 71,60 & $\mathbf{x}$ & & \\
\hline Pano costurado - Estopa & Un & 0,24 & & & $\mathbf{x}$ \\
\hline Correia A 36 & Un & 3,84 & $\mathbf{x}$ & & \\
\hline Correia B 43 & Un & 6,03 & $\mathbf{x}$ & & \\
\hline Correia A 71 & Un & $\mathbf{8 , 0 5}$ & $\mathbf{x}$ & & \\
\hline Enxada & peca & 16,10 & & & $\mathbf{x}$ \\
\hline Correia A 38 & Un & 4,44 & $\mathbf{x}$ & & \\
\hline Rolamento 1208 & Un & 55,24 & $\mathbf{x}$ & & \\
\hline Óleo hidráulico ISO 68 & 1 & 5,50 & & $\mathbf{x}$ & \\
\hline Lâmpada econômica 250 w & peca & 9,87 & & & $\mathbf{x}$ \\
\hline Correia B 35 & Un & 5,74 & $\mathbf{x}$ & & \\
\hline Água sanitária & $\mathbf{I}$ & 1,70 & & & $\mathbf{x}$ \\
\hline \multicolumn{4}{|c|}{ LEGENDA } & & \\
\hline \multirow{3}{*}{\multicolumn{3}{|c|}{$\begin{array}{l}\text { Parada na produção ou prejuizo direto } \\
\text { Redução na produção e/ou Prejuizo Indireto } \\
\text { Não para a produção nem causa prejuizo }\end{array}$}} & C1 & & \\
\hline & & & $\mathrm{C2}$ & & \\
\hline & & & C3 & & \\
\hline
\end{tabular}

Fonte: Pesquisa de campo(2014)

Figura 07 representa uma tabela que foi extraída de uma listagem de material adquiridos durante todo o ano de 2013 a qual foi submetida aos gerentes de produção para determinarem a criticidade dos materiais, considerando os critérios utilizados para avaliação de criticidade, porém, por motivos de limitação de espaço, apenas alguns itens de uma lista de mais de cinco mil itens, foram apresentados. Mesmo nesta amostra da tabela original, é possível perceber que em uma empresa de nutrição animal os materiais comprados são relativamente baratos, contudo, nota-se que mais de 70\%, apresentam criticidade média e alta, justificando assim o uso de estratégias de compras e gestão de estoque.

Já na Figura 8, a seguir, são apresentados, os resultados da classificação ABC dos materiais, utilizados para determinar as estratégias de gestão de estoque da empresa em estudo.

Figura 8: Classificação ABC com criticidade dos materiais da empresa

\begin{tabular}{|c|c|c|c|c|c|c|c|}
\hline Material & Unid. & $\begin{array}{l}\text { Preço } \\
\text { médio }\end{array}$ & Quant & Valor & $\%$ Valor & $\mathbf{A B C}$ & Critic. \\
\hline Gás GLP $20 \mathrm{~kg}$ & Un & 77,00 & 290 & $22.330,00$ & $17,82 \%$ & A & 1 \\
\hline Respirador PFF2 & Un & 0,86 & 15.900 & $13.674,00$ & $10,91 \%$ & A & 2 \\
\hline Papel toalha $6 \times 20 \times 200 \mathrm{mts}$ & rolo & 13,61 & 480 & $6.532,80$ & $5,21 \%$ & A & 3 \\
\hline Sacos de amostras & Un & 0,35 & 15.300 & $5.355,00$ & $4,27 \%$ & A & 2 \\
\hline Redutor M 8 - 1/15 & Un & $1.702,19$ & 3 & $5.106,57$ & $4,08 \%$ & A & 1 \\
\hline Celula de carga modelo $S$ & peca & 689,89 & 4 & $2.759,56$ & $2,20 \%$ & A & 1 \\
\hline Redutor M $10-1 / 15$ & peca & $2.320,55$ & 1 & $2.320,55$ & $1,85 \%$ & A & 1 \\
\hline 1331 - peça máquina de costura & peca & 239,20 & 2 & 478,40 & $0,38 \%$ & $\mathrm{~B}$ & 1 \\
\hline Disco de corte $4.1 / 2^{\prime \prime} \times 1 / 16 \times 7 / 8$ & peca & 3,57 & 125 & 446,25 & $0,36 \%$ & $\mathrm{~B}$ & 2 \\
\hline 1314 - peça máquina de costura & peca & 437,36 & 1 & 437,36 & $0,35 \%$ & B & 1 \\
\hline Touca TNT & peca & 0,09 & 4.800 & 432,00 & $0,34 \%$ & $B$ & 3 \\
\hline Adesivo $\mathrm{p} /$ Correia $1000 \mathrm{~g}$ & frasc & 71,60 & 6 & 429,60 & $0,34 \%$ & $\mathrm{~B}$ & 2 \\
\hline Pano costurado - Estopa & Un & 0,24 & 1.747 & 419,28 & $0,33 \%$ & $\mathrm{~B}$ & 3 \\
\hline Correia A 36 & Un & 3,84 & 30 & 115,20 & $0,09 \%$ & c & 1 \\
\hline Correia B 43 & Un & 6,03 & 19 & 114,57 & $0,09 \%$ & C & 1 \\
\hline Correia A 71 & Un & 8,05 & 14 & 112,70 & $0,09 \%$ & c & 1 \\
\hline Enxada & peca & 16,10 & 7 & 112,70 & $0,09 \%$ & C & 3 \\
\hline Correia A 38 & Un & 4,44 & 25 & 111,00 & $0,09 \%$ & C & 1 \\
\hline Rolamento 1208 & Un & 55,24 & 2 & 110,48 & $0,09 \%$ & $\mathrm{C}$ & 1 \\
\hline Óleo hidráulico ISO 68 & 1 & 5,50 & 20 & 110,00 & $0,09 \%$ & c & 1 \\
\hline Lâmpada econômica 250 w & peca & 9,87 & 11 & 108,57 & $0,09 \%$ & c & 2 \\
\hline Correia B 35 & Un & 5,74 & 18 & 103,32 & $0,08 \%$ & c & 1 \\
\hline Água sanitária & 1 & 1,70 & 60 & 102,00 & $0,08 \%$ & $\mathrm{c}$ & 3 \\
\hline
\end{tabular}

Fonte: Pesquisa de campo(2014) 
$\mathrm{Na}$ proposta de gestão de estoque para o modelo, são tomadas ações estratégicas para cada item de acordo com a sua classificação, onde os materiais de classificação A-1 e A-2, devem ser utilizadas estratégias mais elaboradas de forma a permitir maior controle na sua utilização e otimização dos custos, assim como para todos os materiais com nível de criticidade 1-Alta, que também devem receber atenção especial. Já para os demais níveis de criticidade as estratégias são menos elaboradas e mais flexíveis, de forma a que não se utilize de muitos recursos de gestão de estoque e nem se perca o controle do uso destes materiais.

Outra ferramenta para auxiliar na gestão do estoque, é a realização do inventário, tanto para auxiliar na gestão do estoque, como para cumprimento de exigências legais determinadas pela legislação tributária do país. Para o modelo de gestão de compras proposto neste trabalho, as contagens de materiais devem seguir as boas práticas de inventário estipuladas pelo Conselho Federal de Contabilidade.

\subsubsection{Ferramentas de compra estratégica}

Partindo-se da ideia de que cada empresa estrutura seu departamento de compras de acordo com a percepção de valor atribuído ao setor, é apresentado a seguir, uma proposta de ferramenta estratégica de compras adequada ao perfil de uma empresa de nutrição animal considerando as suas particularidades, propiciando assim, que a empresa deixe de usar práticas obsoletas de compras e passe a utilizar ferramentas mais elaboradas para gerir suas compras.

Para a empresa em estudo, a prática do sistema tradicional de compras ainda era novidade, seu processo antigo era truncado e pouco eficiente, o que gerava transtornos, gastos desnecessários e prejuízos diretos e indiretos, por não ter nem mesmo um processo tradicional de compras funcionando adequadamente, conforme apresentado por Monteiro e Tinoco (2013). Por estes motivos sugere-se o uso de duas ferramentas de compras estratégicas no modelo proposto: a Matriz de Kraljic, (1983) para definição de estratégias de compra para cada item, aliado ao modelo de $\operatorname{Ogden}(2003)$ para definição dos fornecedores mais adequados para empresa.

$\mathrm{Na}$ tratativa dos materiais pela Matriz de Kraljic o impacto financeiro utilizado como critério, é mensurado não no valor financeiro do material como propõe o autor, mas sim no impacto direto que a falta do material ocasiona a produção. Quanto ao risco de fornecimento, adotaram-se os critérios tidos como importantes para a empresa pelo seu perfil, como: a quantidade de fornecedores, qualidade do material, confiabilidade, logística, preço, negociabilidade, e estrutura do fornecedor para atender a demanda. Já com relação aos fornecedores optou-se como estratégia principal, trabalhar com quadro reduzido de fornecedores, como proposto por $\operatorname{Ogden}(2003)$, devido ao perfil da empresa que procura manter um quadro enxuto de funcionários, assim, com menos 
fornecedores para administrar, menos esforço operacional é necessário. Para atender esta estratégia principal, adotou-se os sete passos de Ogden(2003).

Ainda sobre a Matriz de Kraljic, é preciso definir os critérios que serão considerados nas avaliações para atribuir um score para o Impacto Financeiro e o Risco de fornecimento. Considerando o perfil da empresa em estudo existe uma tendência dos critérios para avaliar o risco de fornecimento serem mais relevantes e numerosos do que os critérios para mensurar o impacto financeiro.

Apesar desta tendência, é importante que haja uma equipe multifuncional constituída por técnicos, gestores e até mesmo os diretores da empresa, para julgar com rigor os critérios estabelecidos, e assim determinar adequadamente os pesos dos critérios estabelecidos pela empresa. Pois é através deste julgamento e pontuação de cada item nos critérios avaliados que os materiais são posicionados na Matriz de Kraljic e, consequentemente, adotam-se as estratégias estabelecidas para cada categoria.

Existem ferramentas para determinação dos pesos entre os critérios, que tendem a anular a subjetividade na avaliação da equipe e minimizar erros e tomadas de decisões equivocadas. Entretanto, considerando a experiência do autor na área e conhecimento sobre o contexto da empresa, sugerem-se alguns critérios que servem como norte na implementação da ferramenta. Os critérios sugeridos são apresentados nas Tabelas 2 e 3 a seguir.

Tabela 2: Critérios para determinação do escore do Impacto Financeiro

\begin{tabular}{ll}
\hline \multicolumn{1}{c}{ Critério } & \multicolumn{1}{c}{ Avaliação } \\
\hline $\begin{array}{l}\text { Custo de aquisição do } \\
\text { material }\end{array}$ & Avalia-se o valor de compra do material no mercado. \\
\hline Impacto na produção & $\begin{array}{l}\text { Neste critério deve-se avaliar o impacto ocasionado na produção caso haja a falta do } \\
\text { mesmo, ou se ocasiona redução de produtividade. Quanto maior a influencia na } \\
\text { produção, maior o seu impacto. }\end{array}$ \\
\hline $\begin{array}{l}\text { Custo de manutenção do } \\
\text { estoque }\end{array}$ & $\begin{array}{l}\text { Avalia-se neste caso o custo de ter o material no estoque em grande quantidade e } \\
\text { assim manter o almoxarifado abastecido para garantir que não haja parada na } \\
\text { produção. }\end{array}$ \\
\hline $\begin{array}{l}\text { Impacto da falta de estoque } \\
\text { na produção }\end{array}$ & $\begin{array}{l}\text { O que deve ser avaliado neste critério é o quanto a empresa é prejudicada, caso não } \\
\text { tenha o item no estoque e este é requisitado, ou seja, não ter o produto no estoque } \\
\text { impacta na produção? }\end{array}$ \\
\hline Fonte:Pesquisa de campo (2014)
\end{tabular}

Fonte:Pesquisa de campo (2014) 
Tabela 3: Critérios para determinação do escore do Risco de Fornecimento

\begin{tabular}{cl}
\hline Critério & \multicolumn{1}{c}{ Avaliação } \\
\hline & Avalia-se neste caso, quantos fornecedores a empresa tem a sua disposição, \\
aptos para atender a sua demanda de um determinado material, considera-se que & é preciso ter pelo menos três fornecedores do mesmo material ou substituto \\
direto, para se considerar suprida.
\end{tabular}

Avalia-se qual a qualidade do material fornecido para empresa, para isso deve-se verificar pelo menos os seguintes aspectos: $O$ percentual de peças fornecidas

Qualidade do material oferecido com defeito, o seu acabamento, a sua durabilidade. Também deve-se verifica qual o nível de tecnologia aplicada no material com relação aos outros aplicados a mesma função.

É preciso saber neste critério, quanto o fornecedor é confiável para entregar o material no prazo combinado, se honra seus acordos mantendo uma constância

Pontualidade e confiabilidade na entrega e estrutura de fornecimento de fornecimento, e o quão diversificado são os materiais fornecidos. Também deve-se levar em consideração o quanto o fornecedor pode atender pedidos emergenciais, mantendo o padrão de confiabilidade. É importante verificar também sua prestatividade no atendimento.

\begin{tabular}{ll}
\hline Preço & Avalia-se o preço a ser pago no material. \\
\hline & Também é muito importante avaliar a localização do fornecedor e o quanto isso \\
& favorece no fornecimento, em algumas situações a localização não é fator \\
Logística & determinante para escolha do fornecedor, pois alguns mais distantes são mais \\
& eficientes na entrega do que os mais próximos, entretanto este é um ponto que \\
não pode deixar de ser avaliado.
\end{tabular}

Facilidade de negociação Neste critério deve-se levado em consideração o quão flexível é o fornecedor ao negociar seus preços, prazos de entrega, substituição de material, etc.

Fonte:Pesquisa de campo (2014)

\subsubsection{Validação do Modelo Proposto}

Para validação do modelo, foi realizada uma entrevista com a diretoria administrativa da empresa, responsável pela área baseada nos resultados deste trabalho, que visou propor um modelo de gestão de compras de materiais e serviço, que torna o setor alinhado as estratégias corporativas.

Para tanto o modelo propôs uma reestruturação do processo de compras e de sistema, no intuito de tornar possível a gestão estratégica de compras. Relacionado ao processo, foram apresentados o Diagrama SIPOC, como macro processo de compras; os fluxogramas dos processos, mostrando graficamente as atividades de cada elemento no processo, considerando as etapas do processo de compras apresentadas na literatura. Também foi apresentado à diretoria, a proposta de ferramentas para a gestão de estoque (etapa 10 do modelo proposto), seguido das ferramentas de compra estratégica adaptada às necessidades da empresa.

Após a apresentação, a diretoria aprovou o modelo proposto sem propor adequações. Entretanto, as ferramentas de compra estratégica não serão implantadas em um primeiro momento, pois segundo a diretoria, ainda é preciso que a empresa organize satisfatoriamente seu processo de compra para então ousar em adotar estas ferramentas mais elaboradas na gestão de suas compras e conseguir melhorias nos seus resultados.

O restante do modelo já se encontra em fase de implantação na empresa. Sendo assim, foi adquirido um novo software ERP ( EnterpriseResource Planning) cujoas parametrizações do 
sistema relacionadas a compras, foram todas realizadas com base no processo proposto neste trabalho, e então foi possível verificar que o sistema atendia completamente todos os requisitos que o modelo considerou como importante um software de gestão de compras.

A previsão é que todo projeto de implantação do novo sistema ERP com todos os módulos de gerenciamento na empresa leve em torno de nove meses, e para o setor de compras especificamente, são previstas trinta e duas horas para implantação, contemplando as parametrizações do sistema de acordo com os procedimentos proposto neste trabalho, testes, validação e treinamento dos usuários. Acredita-se pelos resultados práticos deste trabalho que brevemente a empresa manifestará o interessa na implantação das ferramentas de compras estratégicas propostas neste modelo de gestão de compras, a Matriz de Kraljic (1983) aliada ao modelo de Ogden (2003).

\section{Conclusões}

Procurou-se com este trabalho propor um modelo de gestão para o setor de materiais e serviços alinhado ás estratégias da empresa segundo análise do sistema de atual de gestão apresentado no trabalho de Monteiro e Tinoco (2013). Para tanto, realizou-se: (i) Levantamento dos principais modelos de gestão estratégica de compras de materiais e serviços existentes na literatura. (ii) Verificação dos objetivos estratégicos e o alinhamento do processo de compras, devido à mudança na diretoria da empresa; (iii) Estruturação do modelo de gestão de compras da empresa em estudo; (iv) Validação do modelo proposto com a alta diretoria da empresa.

O modelo proposto no trabalho compreende o macro processo de gestão de compras (diagrama SIPOC), que proporcionou a construção do fluxograma do processo baseado, a descrição das necessidades estratégicas em cada uma das etapas que são: (i) Solicitações de compras; (ii) Recebimento da solicitação de compras; (iii) Planejamento de compra; (iv) Solicitação de cotações; (v) Determinação do preço; (vi) Emissão da Ordem de Compra (OC); (vii) Recepção e aceitação da mercadoria e (viii) Finalização da compra e processamento da Nota Fiscal; (ix) Contratação de serviços e (x) Gestão de estoque de materiais.

Com este trabalho foi possível então, proporcionar o alinhamento do setor de compras de materiais e serviços da empresa as suas estratégias, a partir da aquisição de um software adequado a gestão de compras, conforme sugerido neste trabalho; da definição clara das atividades de compras no processo, mostrada através dos fluxogramas. O modelo proposto neste trabalho foi melhor documentado de forma a gerar o manual de compras da empresa, o qual foi utilizado para treinar os colaboradores envolvidos no processo de compras. Atualmente, o modelo está sendo implementado parcialmente nas aquisições de materiais e serviços da empresa. As ferramentas de compras 
estratégicas ainda não foram utilizadas, possivelmente pelo grau de maturidade da empresa em relação ao seu uso.

Sendo assim, dentre as sugestões para dar continuidade a este trabalho, recomenda-se a implementação total do modelo na empresa, o acompanhamento do desempenho do modelo através de indicadores financeiros da empresa e a implementação do modelo no setor de compras de materiais produtivos, após as devidas alterações.

\section{Abstract}

An evolution in the concepts and the importance of the supply sector within companies has been noted in the management literature of the supply chain. These new concepts and this change of vision for this sector within organizations, led to the development of this study. The objective of this study was to analyze the purchasing process in a Brazilian company, animal nutrition, to check the alignment of the materials sector management model and services with the company's strategies. For this, it was carried out a mapping of the material purchasing process and services company, where the whole process was followed during six months and a semi structured interview with the company's management responsible for the area in order to verify the alignment of process with the company's strategies. This study can be seen that, conceptually, the management model used by the materials and services sector, is aligned with business strategies, but the infrastructure provided, the process and the staff need to be improved to be aligned with the required strategically.

Key-word: process;purchase;purchase management

\section{Referências}

ARNOLD, J. R. T. Administração de materiais: Uma introdução. 1. ed. Tradução, Celso Rimoli, Lenita R. Rsteves. São Paulo: Atlas, 1999.

BAILY, P.; FARMER, D.; JESSOP, D.; JONES, D. Compras: Princípios e administração. 8. ed. Tradução Ailton Bomfim Brandão. São Paulo: Atlas, 2000.

BALLOU, Ronald H. Gerenciamento da cadeia de suprimentos: logística empresarial. Bookman, 2006.

BARNEY, J. B. Purchasing, supply chain management and sustained competitive advantage: The relevance of resource-based theory.JournalofSupply Chain Management, v. 48, n. 2, p. 3-6, 2012.crossref

BOWERSOX, D. J.; CLOSS, D. J. LogísticaEmpresarial, O Processo de Integração da Cadeia de Suprimento.São Paulo, Editora Atlas, 2009.

BRAGA, A. Evolução estratégica do processo de compras ou suprimentos de bens e serviços nas empresas. Instituto Coppead, Universidade Federal do Rio de janeiro, Rio de Janeiro, 2006.

ANDRADE, G. C. Gestão estratégica de suprimento em uma empresa do setor elétrico brasileiro. 2012. Tese de Doutorado. Universidade Federal do Rio de Janeiro.

SOUZA, A. M. S. T.; SCAVARDA, L. F.; SCAVARDA, A. J. Compras de Itens-não-críticos numa empresa de petróleo. Revista de Administração FACES Journal, v. 7, n. 4, 2008.

DIAS, M.; COSTA, R. F. Manual do comprador: conceitos, técnicas e praticas indispensáveis em um departamento de compras. São Paulo: Edicta, 2003.

DOBLER, D. W.; BURD, D. N. Purchasing and supply management:text and cases. 6. ed. New York: McGrawHill, 1996. 
ELlRAM, L. M.; COOPER, M. C. Supply Chain Management: It's All About the Journey, Not the Destination. Journal of Supply Chain Management, v. 50, n. 1, p. 8-20, 2014.crossref

FERREIRA, L. M.; KHARLAMOV, A. A. Application of Kraljic's Purchasing portfolio matrix in construction industry-A case study. In: International Conference of Industrial Engineering and Operations Management. 2012.

GAITHER, N.; FRAZIER, G. Administração da Produção e Operações.São Paulo: Pioneira Thomson Learning, 2001.

GELDERMAN, C. J.; MAC DONALD, D. R. Application of Kraljic's Purchasing Portfolio Matrix in an Undeveloped Logistics Infrastructure: The Staatsolie Suriname Case. Journal of Transnational Management, v. 13, n. 1, p. 77-92, 2008.

GELDERMAN, K.; VAN WEELE, A. Advancements in the use of a purchasing portfolio approach: A case study. In: Proceedings of the 10th International IPSERA Conference. 2001. p. 403-415. crossref

JOHnSEn, T. E.; HOWARD, M.; MIEMCZYK, J. Purchasing and Supply Chain Management: A Sustainability Perspective.Routledge, 2014.

KIBBELING, M. I. Creating Value in Supply Chains: Supplier's Impact on Value for Customers, Society and Shareholders. Beta PhD Thesis Series, Eindhoven Technology University, Eindhoven, the Netherlands, 2010.

KRALJIC, Peter. Purchasing must become supply management. Harvard business review, v. 61, n. 5, p. 109-117, 1983.

KLIPPEL, M. et al. Matriz de posicionamento estratégico de materiais: conceito, método e estudo de caso. Gestão e Produção, v. 14, n. 1, p. 181-192, 2007.crossref

LAMBERT, D. M.; COOPER, M. C.; PAGH, J. D. Supply chain management: implementation issues and research opportunities. The International Journal of Logistics Management, v. 9, n. 2, p. 1-19, 1998.crossref

LIEW, J. G. L. Classify me!: A Kraljic Portfolio Matrix Exercise. 2014.

MONCZKA, R.; HANDFIELD, R.; GIUNIPERO, L.; PATTERSON, J. Purchasing and supply chain management.Cengage Learning, 2011

MONTEIRO, A.; TINOCO, M. A. C. Análise do Sistema de Gestão de Compras de Materiais e Serviços para uma Empresa de Nutrição Animal: Um Estudo de Caso.Ingeniería Industrial. Actualidad y NuevasTendencias, n. 10, p. 67-74, 2013.

MOREIRA, S. M. da R. Definição de estratégias para a gestão de compras: estudo de caso. 2013.

OGDEN, J. Supply Base Reduction Within Supply Base Reduction. PRACTIX-Good Practices in Purchasing and Supply Chain Management, v. 6, 2003.

SAMPSON, S. E.; SPRING, M. Customer roles in service supply chains and opportunities for innovation.Journal of Supply Chain Management, v. 48, n. 4, p. 30-50, 2012.crossref

SLACK, N. et al.Administração da produção. EdiçãoCompacta. SãoPaulo: Atlas, 1999.

VIANA, J. J. Administração de materiais: um enfoque prático. 1. ed. São Paulo: Atlas, 2002.

WANKE, P. Gestão de estoques na cadeia de suprimentos: decisões e modelos quantitativos. $3^{\text {a }}$ ed. São Paulo: Atlas, 2011.

WEELE, A. J.; RAAIJ, E. M.The future of purchasing and supply management research: About relevance and rigor. Journal of Supply Chain Management, v. 50, n. 1, p. 56-72, 2014.crossref

YIN, R. K. Case Study Research: Design and Methods.SagePublications Inc., USA, 1989. 


\section{Dados do autor}

Nome completo:Alexsandro Monteiro

Mestre em Engenharia de Produção pela UFRGS

Função ou cargo ocupado:Coordenador de materiais e serviços na empresa Novanis Animal LTDA

Endereço completo para correspondência: Rua Diogo Podesta Albres, 454, Cidade Salmen,

Rondonópolis - MT, CEP: 78705-225

Telefones para contato:66 21010645 / 6692050562

e-mail:alexmonteiro9984@gmail.com

Nome completo:María Auxiliadora Canarosso Tinoco

Doutoraem Engenharia de Produção pela UFRGS

Filiação institucional: Universidade Federal do Rio Grande do Sul

Departamento: Engenharia de Produção e Transportes

Função ou cargo ocupado: Professor Adjunto

Endereço completo para correspondência: Av. Osvaldo Aranha, 99, 5to andar. CEP: 90035-190

Telefones para contato: 51-33083490

e-mail:maria@producao.ufrgs.br

Submetido em: 12-04-2015

Aceito em: 14-09-2015 